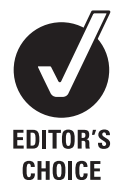

Department of Anaesthesiology, Universitaetsklinikum Hamburg-Eppendorf, Germany

\section{Correspondence to} Stefan Maisch, Department of Anaesthesiology, University Hospital Hamburg-Eppendorf Martinistrasse 52, 20246 Hamburg, Germany: smaisch@gmx.de

Accepted 23 August 2010 Published Online First 14 October 2010

\title{
Comparison of the over-the-head, lateral and alternating positions during cardiopulmonary resuscitation performed by a single rescuer with a bag-valve-mask device
}

\author{
Stefan Maisch, Eike Gamon, Alexander llisch, Alwin E Goetz, Gunter N Schmidt
}

\section{ABSTRACT}

Background The 2005 guidelines for cardiopulmonary resuscitation (CPR) do not include a statement on performance of basic life support by a single healthcare professional using a bag-valve-mask device. Three positions are possible: chest compressions and ventilations from over the head of the casualty (over-the-head CPR), from the side of the casualty (lateral CPR), and chest compressions from the side and ventilations from over the head of the casualty (alternating CPR). The aim of this study was to compare CPR quality of these three positions.

Methods 102 healthcare professionals were randomised to a crossover design and performed a 2-min CPR test on a manikin for each position.

Results The hands-off time over a 2-min interval was not significantly different between over-the-head (median $31 \mathrm{~s}$ ) and lateral (31 s) CPR, but these compared favourably with alternating CPR (36 s). Over-the-head CPR resulted in significantly more chest compressions (155) compared with lateral (152) and alternating CPR (149); the number of correct chest compressions did not differ significantly (119 vs 122 vs 109). Alternating CPR resulted in significantly less inflations (eight) compared with over-the-head (ten) and lateral CPR (ten). Lateral CPR led to significantly less correct inflations (three) compared with over-the-head (five) and alternating CPR (four).

Conclusions In the case of a single healthcare professional using a bag-valve-mask device, the quality of over-the-head CPR is at least equivalent to lateral, and superior to alternating CPR. Because of the potential difficulties in bag-valve-mask ventilation in the lateral position, the authors recommend over-the-head CPR.

\section{INTRODUCTION}

The guidelines for basic life support (BLS) published by the European Resuscitation Council ${ }^{1}$ and the International Liaison Committee on Resuscitation ${ }^{2}$ describe one-rescuer cardiopulmonary resuscitation (CPR) for a lay rescuer without the use of equipment, and two-rescuer CPR for two healthcare professionals with the use of equipment such as a bag-valve-mask device. The use of a bag-valvemask device has three major advantages: the possibility of administering supplementary oxygen (up to $100 \%$ for bag-valve-mask devices with demand valve), avoidance of transmission of infections (such as tuberculosis ${ }^{3}$ or severe acute respiratory distress syndrome ${ }^{4}$ ) and, presumably, a reduction in rescuer fatigue. The guidelines do not contain any statement with regard to a single rescuer using a bag-valvemask device. There are three possibilities for performing CPR by a single rescuer using a bag-valve-mask device:

- Over-the-head CPR: performance of chest compressions and ventilations from over the head of the casualty according to the guidelines for CPR in confined spaces.

- Lateral CPR: performance of chest compressions and ventilations from the side of the casualty according to the guidelines for one-rescuer CPR for a lay rescuer with mouth-to-mouth ventilation.

- Alternating CPR: performance of chest compressions from the side of the casualty and ventilations from over the head of the casualty according to the guidelines for CPR for two healthcare professionals.

A few studies of one-rescuer over-the-head CPR have been reported. ${ }^{5-11}$ The results of these studies are controversial, and they were performed according to the 2000 guidelines, ${ }^{12}$ in which the compression-ventilation ratio was 15:2, compared to $30: 2$ according to the 2005 guidelines. Change of position (side to head) has never been studied.

The purpose of this study was to analyse, on a manikin, the efficacy of three CPR positions performed by a single rescuer using a bag-valvemask device and to compare CPR quality of the single-rescuer positions with standard dualoperator CPR. Such a situation where a single rescuer equipped with a bag-valve-mask device is present and the victim is lying on the floor is possible in a prehospital scenario or at the hospital with a patient collapsed out of bed. Another scenario for over-the-head CPR technique is a cardiac arrest situation with two rescuers at a scene, with one rescuer beginning CPR alone while the other performs additional tasks.

\section{METHODS}

\section{Participants and setting}

Paramedics and emergency medical technicians (EMT) from two rural districts of Greater Hamburg, Germany, were asked to participate in the study in the course of obligatory annual education and training; all participants in the training agreed to take part in the study. The local ethics committee declared that such a study did not require ethical approval. All participants gave written informed consent for their CPR performance data to be evaluated. 


\section{Study design}

The study was a randomised crossover design. Randomisation was performed using a list of random numbers generated by Microsoft Excel V11.3.7.; random numbers were allocated to the five different methods and positions in equal shares: overthe-head CPR, lateral CPR, alternating CPR, ventilation during dual-operator CPR and chest compression during dual-operator CPR. Participants received a short, standardised, theoretical introduction concerning the different methods of single-rescuer CPR, which lasted for about 15 min and included the demonstration of the different positions of single-rescuer CPR. Afterwards, each participant practiced CPR in the three positions for about $15 \mathrm{~min}$ on two CPR manikins (Resusci Anne Simulator, Laerdal Medical AS, Stavanger, Norway). Theoretical introduction and training for dual-operator CPR was not necessary because this method was well known to all participants. For over-the-head CPR, instructions were given for the rescuer to kneel with the head of the manikin between his or her knees/ thighs and to place the hands in the centre of the chest consistent with guidelines for $\mathrm{BLS}^{1}$; the heel of the hand was placed across the sternum (see figure 1). Chest compressions were performed at a rate of approximately $100 / \mathrm{min}$, with an audio prompt helping to maintain the frequency; this audio prompt was used for all CPR positions. Participants were shown how to perform bag-valve-mask ventilation from the lateral position (see figure 2). The technique of alternating positions was a mixture of both techniques described: lateral compression and ventilation from over the head. The optimum tidal volume was defined as 400-600 ml; this was according to the guidelines, which recommend $6-7 \mathrm{ml} / \mathrm{kg}$ body weight and the presumed weight of $70-80 \mathrm{~kg}$ of the simulator. Thus, the value of $400-600 \mathrm{ml}$ is rounded from 420 to $560 \mathrm{ml}$. Ventilation was administered with a bag-valve-mask device (Laerdal Silicone Resuscitator ${ }^{\circledR}$ with an Adult Disposable Resuscitator mask size 4, Laerdal Medical AS, Stavanger, Norway).

Study participants were informed about the general aim of the study but were blinded to the outcome and the stated hypothesis. Each participant performed 30 chest compressions and two breaths, alternately, with a bag-valve-mask for 2 min in the over-the-head, lateral and alternating positions alone, and in each dual-operator CPR position in a randomised order on the simulator (Resusci Anne Simulator, Laerdal Medical AS, Stavanger, Norway). The simulator was placed on the floor. Between the two different positions, participants had at least

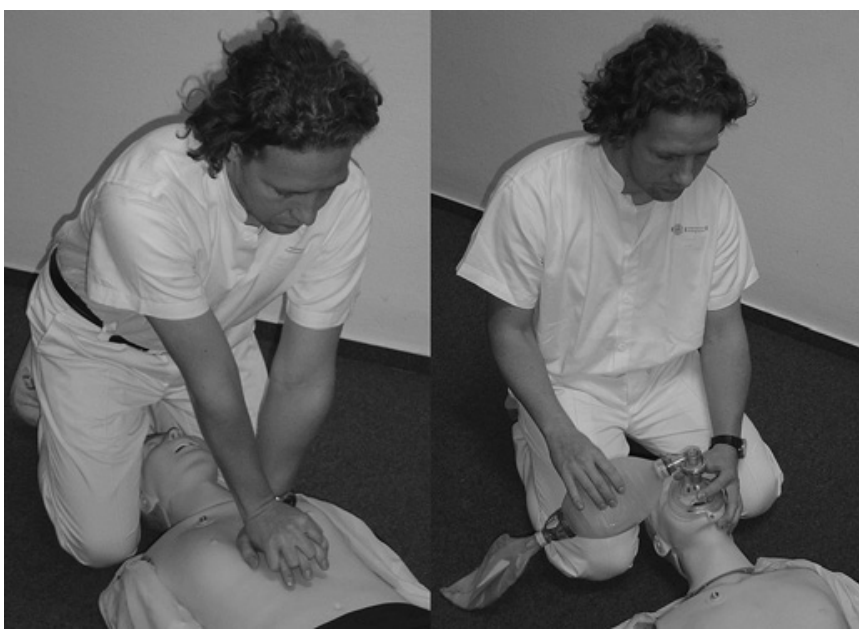

Figure 1 Over-the-head cardiopulmonary resuscitation (CPR) position for chest compressions and ventilation.

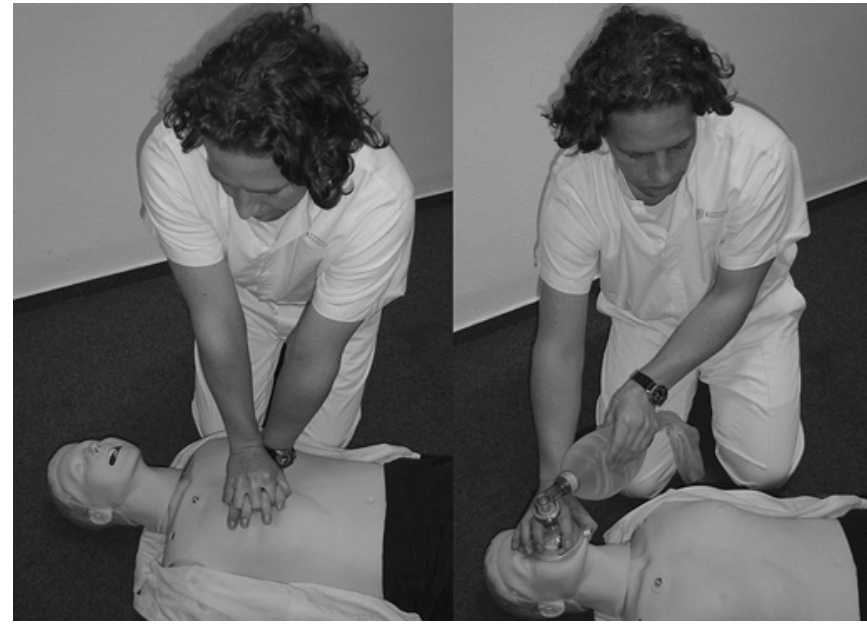

Figure 2 Lateral cardiopulmonary resuscitation (CPR) position for chest compressions and ventilation.

a 30-min break for recreation, to exclude diminished quality because of exhaustion.

The simulator was connected to a computer running analysis software (Laerdal PC SkillReporting System ${ }^{\circledR}$ software, version 2.21, Laerdal Medical AS, Stavanger, Norway). The following data were recorded during a 2-min period of BLS: 'hands-off time' (interruption of chest compressions), time for two ventilations, number of chest compressions, compression rate per min, compression depth, hand position, release, duty cycle (time spent compressing the chest as a proportion of the time between the start of one cycle of compression and the start of the next), number of ventilations, ventilation volume, duration of inflation and inspiratory flow rate. The SkillReporting system measures hands-off time and quality of chest compression parameters. Ventilation parameters were registered by the SkillReporting System; volume curves were analysed offline to determine tidal volume and number of inflations. The chest compression and ventilation parameters were classified as correct or incorrect, according to the published 2005 guidelines of the European Resuscitation Council ${ }^{12}$ : correct compression rate per min (90-110/min), correct compression depth (40-50 mm), correct hand position (automatically recorded by device), correct release (complete release of pressure after each compression), correct ventilation volume $(400-600 \mathrm{ml})$ and correct ratio of compressions to ventilations (30:2). Incorrect compressions could result from one or more simultaneous errors, for example, incorrect hand position and chest compression that was too deep.

The primary outcome was the hands-off time during the 2 -min test interval. The secondary outcome was the number of chest compressions and ventilations during the 2-min test interval.

After taking part in the study, participants had to fill out a questionnaire to evaluate their practical experience in CPR and the used and preferred single-rescuer CPR method.

\section{Statistical analysis}

According to Hüpfl et $a l,{ }^{10}$ it was determined that 99 participants were required to have an $80 \%$ chance of detecting as significant (at the two-sided 5\% level) a 5\% difference between standard single-rescuer and over-the-head CPR in the mean hands-off time required for two ventilations.

All data were analysed using the statistics program SPSS 11.5. Non-normally distributed data were analysed using Friedman's test to reduce the significance level and post hoc by the 
Wilcoxon signed rank test, and are presented as total number or median (with IOR). p Values $<0.05$ are considered statistically significant.

\section{RESULTS}

There were 102 paramedics and EMTs (19 female, 83 male) who participated in the study. No participants were excluded from enrolment or analysis. The demographics of participants were as follows: the median age was 26 years (range 18-62), average height was $181 \mathrm{~cm}$ (range 162-195) and average weight was $80 \mathrm{~kg}$ (range 51-128). Sixty-nine of the participants were paramedics; 33 of the participants were EMTs. They had worked in the emergency medical services for a median of 6 years (range $0-38$ ). All participants had practical experience in CPR including the use of bag-valve-mask ventilation; the participants had performed on average $42 \mathrm{CPRs}$ in the past (range 1-500). Ninety-two of the participants $(90 \%)$ had performed over-thehead CPR

The results of the compression and ventilation parameters are shown in table 1 and figure 3.

In a comparison of the three single-rescuer positions, it was found that the total hands-off time during a 2-min period using over-the-head CPR (median $31 \mathrm{~s}$ ) was comparable to that of lateral CPR (31 s) and significantly shorter than that of alternating CPR $(36 \mathrm{~s})$. There were significantly more chest compressions during a 2-min period using over-the-head CPR (155) compared with lateral (152) and alternating CPR (149). The number of correct chest compressions did not differ significantly (119 with over-the-head CPR vs 122 with lateral CPR vs 109 with alternating CPR). The number of ventilations during a 2-min period using over-the-head CPR (median ten) was comparable to that of lateral CPR (ten) and significantly higher than that of alternating CPR (eight). Significantly more correct ventilations were performed during a 2-min period using over-the-head (median five) or alternating CPR (four) compared with lateral CPR (three).

Comparing the single-rescuer CPR methods with the standard dual-operator CPR, it was found that the total hands-off time during a 2-min period using dual-operator CPR (median $18 \mathrm{~s}$ ) was significantly shorter than using single-rescuer CPR. There were significantly more chest compressions (median 180) and correct chest compressions (148) during a 2-min period using dual-operator CPR compared with single-rescuer CPR. Significantly more ventilations were performed during a 2 -min period using dual-operator CPR (median ten) compared with singlerescuer $\mathrm{CPR}$, whereas the number of correct ventilations did not differ significantly between dual-operator CPR (median four) compared with over-the-head CPR (five) and alternating CPR (four); when compared solely with lateral CPR, dual-operator CPR led to significantly more correct ventilations (four versus three).

There were no significant differences between female and male participants.

The preferred CPR technique for a single rescuer performing CPR with a bag-valve-mask device was $86.3 \%$ for over-thehead CPR, $7.8 \%$ for lateral CPR and $2.9 \%$ for alternating CPR; $2.9 \%$ had no preferred position.

\section{DISCUSSION}

The principal finding of this study was that the quality of CPR performed using a bag-valve-mask device by one single rescuer performing over-the-head CPR was superior to lateral or alternating CPR. Regarding the shorter hands-off time, the number and quality of chest compressions and the number of

Table 1 Efficacy of the different cardiopulmonary resuscitation (CPR) positions for chest compression and ventilation during a 2-min CPR test

\begin{tabular}{|c|c|c|c|c|c|}
\hline & Dual operator CPR & Over-the-head CPR & Lateral CPR & Alternating CPR & Significance \\
\hline \multicolumn{6}{|l|}{ Chest compressions } \\
\hline Number of chest compressions & $180(175-185)$ & $155(150-161)$ & $152(149-161)$ & $149(139-153)$ & $\neq ， \S ， q, * * ， † \dagger ， \neq \ddagger$ \\
\hline Rate of compressions (per min) & $106(105-111)$ & $106(105-110)$ & $106(104-109)$ & $106(105-111)$ & \\
\hline Chest compressions correct & $148(84-171)[82.2 \%]^{*}$ & $119(96-142)[76.8 \%]^{*}$ & $122(74-146)[80.3 \%]^{*}$ & $109(89-131)[73.2 \%]^{*}$ & $\neq, \S$, 甲 \\
\hline Depth $40-50 \mathrm{~mm}$ & $163(127-175)[90.6 \%]^{*}$ & $139(116-149)[89.7 \%]^{*}$ & $141(112-149)[92.8 \%]^{*}$ & $127(109-143)[85.2 \%]^{*}$ & $\ddagger, \S$, ब \\
\hline Depth $<40 \mathrm{~mm}$ & $7(1-29)[3.9 \%]^{*}$ & $3(1-19)[1.9 \%]^{*}$ & $4(1-13)[2.6 \%]^{*}$ & $5(1-16)[3.4 \%]^{*}$ & ब \\
\hline Depth $>50 \mathrm{~mm}$ & $1(0-8)[0.6 \%]^{*}$ & $1(0-21)[0.6 \%]^{*}$ & $3(0-12)[2.0 \%]^{*}$ & $1(0-15)[0.7 \%]^{*}$ & \\
\hline Correct hand position & $179(172-184)[99.4 \%]^{*}$ & $150(134-156)[96.8 \%]^{*}$ & $150(139-154)[98.7 \%]^{*}$ & $143(125-150)[96.0 \%]^{*}$ & 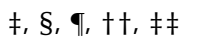 \\
\hline Complete recoiling & $177(167-182)[98.3 \%]^{*}$ & $150(144-158)[96.8 \%]^{*}$ & $150(145-155)[98.7 \%]^{*}$ & $142(130-150)[95.3 \%]^{*}$ & 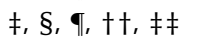 \\
\hline Depth of chest compressions (mm) & $43(41-45)$ & $44(42-48)$ & $44(42-47)$ & $44(42-46)$ & $\neq, \S, \uparrow$ \\
\hline
\end{tabular}

\section{Inflations}

Number of inflations

Tidal volume $400-600 \mathrm{ml}$

Tidal volume $<400 \mathrm{ml}$

Tidal volume $>600 \mathrm{ml}$

Tidal volume $(\mathrm{ml})$

$$
\begin{aligned}
10 & (10-12) \\
4 & (2-7)[40.0 \%] \dagger \\
4 & (1-8)[40.0 \%] \dagger \\
0 & (0-2)[0.0 \%] \dagger \\
424 & (342-522)
\end{aligned}
$$

$$
\begin{aligned}
10 & (8-10) \\
5 & (1-6)[50.0 \%] \dagger \\
4 & (1-7)[40.0 \%] \dagger \\
0 & (0-2)[0.0 \%] \dagger \\
415 & (279-507)
\end{aligned}
$$

$$
\begin{aligned}
10 & (8-10) \\
3 & (0-5)[30.0 \%] \dagger \\
6 & (3-8)[60.0 \%] \dagger \\
0 & (0-0)[0.0 \%] \dagger \\
345 & (218-425)
\end{aligned}
$$

$$
\begin{aligned}
8 & (8-10) \\
4 & (2-6)[50.0 \%] \dagger \\
3 & (1-7)[37.5 \%] \dagger \\
0 & (0-1)[0.0 \%] \dagger \\
401 & (293-493)
\end{aligned}
$$

\footnotetext{
Hands-off time

Total hands-off time (s)

$3.3(2.7-3.8)$

$18(15-20)$ *Percentage refers to total number of chest compressions.

†Percentage refers to total number of inflations.

$\neq \mathrm{p}<0.05$ dual operator versus over-the-head position.

$\S p<0.05$ dual operator versus lateral position.

$\mathbf{9}<0.05$ dual operator versus alternating position.

$*^{* *} \mathrm{p}<0.05$ lateral versus over-the-head position.

$\dagger+p<0.05$ alternating versus over-the-head position.

$\neq \neq p<0.05$ lateral versus alternating position.
}

$14.6(12.5-16.7)$

$\begin{aligned} 6.8 & (6.2-7.5) & 7.3 & (6.2-7.8) \\ 31 & (28-34) & 31 & (29-34) \\ 25.8 & (23.3-28.3) & 25.8 & (24.2-28.3)\end{aligned}$

$8.8(7.1-10.0)$
$36(32-41)$
$30.0(26.9-34.0)$

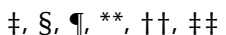

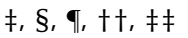

$\ddagger$ §, 凤, †十, キキ

The term 'rate of compressions' refers to the frequency and not to the number of chest compressions. Data are shown as median (first and third quartile). $\mathrm{p}<0.05$ was considered significant. 
Figure 3 Comparison of total handsoff time, number of chest compressions per min and number of inflations per min of the different cardiopulmonary shown as box plots with median, $75 \%$ and $25 \%, 90 \%$ and $10 \%$, and $95 \%$ and $5 \%$ percentiles. $+=p<0.05$ between different positions. resuscitation (CPR) positions. Values are
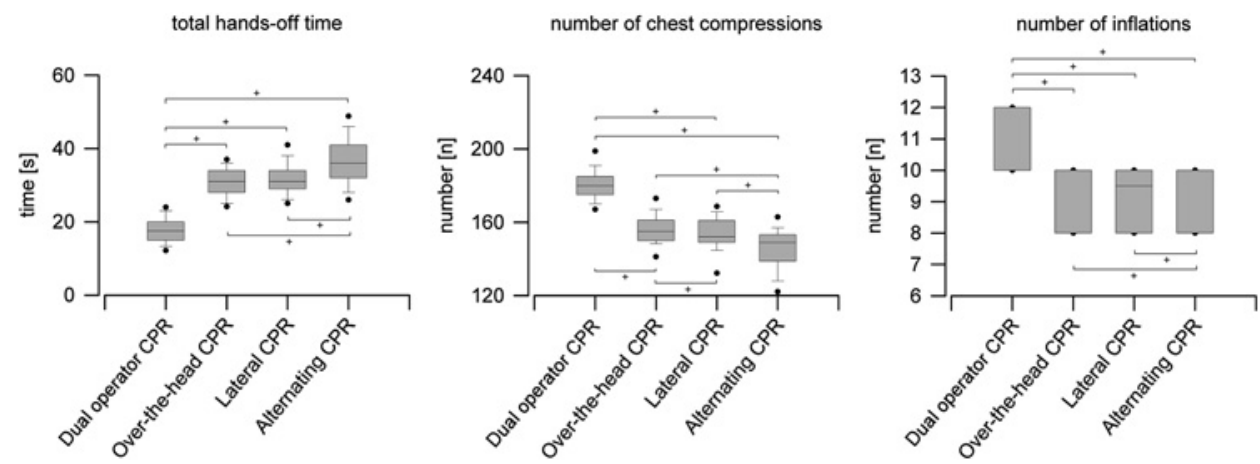

ventilations over the head and lateral CPR performed on a simulator using a bag-valve-mask device were significantly superior to alternating CPR. Concerning the quality of ventilations, over-the-head CPR resulted in significantly more correct ventilations compared with lateral CPR. The quality of ventilations with regard to correct tidal volume during lateral CPR was significantly worse compared with the other CPR positions.

Dual-operator CPR was significantly superior to all singlerescuer methods regarding the hands-off time, the number and quality of chest compressions, and the number of ventilations.

Although the study was powered to detect a $5 \%$ difference in performance, there are no data to show whether this is clinically significant. Thus, some statistically significant differences might be of no clinical relevance. In some cases, for example number of inflations, the detected statistical significance between alternating and over-the-head CPR and between lateral and alternating $\mathrm{CPR}$ is very unlikely to be clinically significant.

Potential scenarios for the over-the-head CPR technique are as follows: one rescuer who is alone at a scene, or two rescuers in a cardiac arrest situation with one rescuer beginning CPR alone while the other performs additional tasks.

A few other studies have evaluated the quality of over-thehead CPR. ${ }^{5-11}$ Gliwitzky et al and Wolcke et $a l^{5-7}$ compared over-the-head CPR with standard two-rescuer CPR in an advanced life support scenario. In contrast to the present findings, these investigators found a significantly lower number of delivered chest compressions in the over-the-head group, but no significant difference in quality of chest compressions and ventilations between the two techniques.

Handley and Handley compared over-the-head CPR performed in a confined space with standard CPR, both as one-person and two-person methods. ${ }^{8}$ The authors focused on trained laypersons who had no previous experience with overthe-head CPR. In opposition to the present results, there was no difference between the two methods, except for more frequent wrong hand positioning in the over-the-head CPR group (30.4\% vs $7.7 \%$ in the standard CPR group). The quality of ventilation, which was performed using a pocket mask, was not assessed in this trial.

Perkins et al compared over-the-head CPR and standard CPR in a group of BLS instructors who had no previous experience with over-the-head CPR. ${ }^{9}$ Ventilation was performed using a pocket mask. Contrary to the present findings, there was no difference in quality of chest compression and ventilation between the two techniques, with the exception that hand positioning during chest compressions was better in the over-the-head group (incorrect compressions 76 vs 300 in the standard group).

Hüpfl et al compared over-the-head CPR with a self-inflating bag, with standard CPR with mouth-to-mouth ventilation in professional medical personnel experienced in bag-valve-mask ventilation and in over-the-head CPR. ${ }^{10}$ Comparable to the present results, over-the-head CPR with the use of a selfinflating bag provides superior ventilation, though this technique was compared to standard CPR with mouth-to-mouth ventilation. The quality of chest compressions did not differ between the two study groups.

Bollig et al compared over-the-head CPR with bag-valvemask ventilation to lateral CPR with bag-valve-mask ventilation in an advanced cardiac life support scenario performed by paramedic students, who were experienced in standard CPR but not in over-the-head CPR. ${ }^{11}$ In both experiments one rescuer delivered chest compressions and ventilations while the other rescuer performed additional tasks. In contrast to the present results, the authors found no differences in ventilation or compression variables or any time factors.

All the studies cited above were performed using the 2000 guidelines. ${ }^{12}$ Therefore, the results might differ if studies were performed using 2005 guidelines with greater emphasis on chest compressions.

According to the present data, over-the-head CPR should be recommended for a single rescuer experienced in bag-valvemask ventilation. This technique led to a significantly shorter hands-off time compared with alternating CPR. The significant difference in the hands-off time of the 2-min interval might be of clinical relevance, as evidence exists that a difference in handsoff time of even less than $10 \mathrm{~s}$ could be detrimental. ${ }^{13}$ Compared with lateral CPR, over-the-head CPR had only a few advantages concerning the ventilation. Although it was demonstrated in the present study that the use of a bag-valve-mask device from the side is possible, it resulted in a worse quality of ventilation. The authors suppose that ventilation with a self-inflating bag from the side of a patient is less practicable than from the side of the simulator. For the combination of chest compression and ventilation, over-the-head CPR evidenced statistical advantages; the authors are convinced that there is a significant clinical relevance for this. The practicability of the over-the-head CPR technique was supported by the fact that the majority of participants preferred this position. Beside the advantages of over-the-head CPR for a single rescuer resulting in the use of the bag-valve-mask device, there are two other reasons for favouring this technique: no movement of the rescuer's body position is needed, and this technique could be used in a confined space with difficult access from the side.

According to the present data, dual-operator CPR resulted in a significantly better CPR quality compared with the singlerescuer CPR positions. Although the median number of inflations of dual operator, over-the-head and lateral CPR was the same, comparison of values of all participants showed a significant difference (please note that median is presented, not mean). 
Only dual-operator CPR met the recommended target of approximately two ventilations per $4 \mathrm{s.}^{1}$

In the 2005 guidelines, achieving a high quality of chest compression, especially compression rate and depth, is considered of utmost significance. ${ }^{2}{ }^{14-16}$ Experimental studies have shown that the delivery of more than 80 compressions per min is necessary for the patient's survival in prolonged cardiac arrest. ${ }^{13}$ Related to the compression rate, another very important aim during CPR is to minimise interruptions in chest compressions, which have a detrimental effect on survival. ${ }^{1} 1718$ In contrast to the present data, hands-off time was $28.5 \%$ in a dual-operator CPR simulator study, in which medical students performed CPR with chest compressions and mouth-to-mouth ventilation for 4 min according to the 2005 guidelines. ${ }^{19}$ This is much longer than the hands-off time of $14.6 \%$ in the present study. In a study by Roessler et al, ${ }^{20}$ in which single-operator CPR with mouth-to-mouth ventilation was performed for $5 \mathrm{~min}$ according to the 2005 guidelines, the hands-off time was $33.3 \%$ in the group of healthcare professionals compared with $25.8 \%$ in the present study. The reasons for this difference could be different practical experience (medical students or physiotherapists, nurses, and physicians compared to paramedics and EMTs in the present study) and method of ventilation (mouth-tomouth ventilation versus bag-valve-mask ventilation in the present study).

\section{Limitations}

Several limitations of the study have to be taken into account when the present findings are extended to a clinical situation. First, the study was performed on a resuscitation simulator, which allows consistent and reproducible experimental conditions with valid results. On the other hand, this does not render any differences in casualty size, chest wall compliance or ease of mask-face seal. Second, it was impossible to blind participants to which technique they were performing; for this reason the order of different positions was randomised. Third, the manikin was positioned on the floor. This is a probable situation in a prehospital setting, but a rare in-hospital scenario.

\section{CONCLUSION}

It has been demonstrated in a simulated CPR model that overthe-head CPR can significantly improve the performance of BLS of a single healthcare professional with a bag-valve-mask device, compared with lateral and alternating CPR. In the case of a casualty requiring CPR and a single rescuer equipped with a bag-valve-mask device, over-the-head CPR can be recommended as superior technique if the rescuer is trained in this method. At any rate, wherever applicable, dual-operator CPR should be the aim, because the quality of BLS performed by two rescuers is significantly better compared with any single-rescuer CPR method.
Acknowledgements The authors wish to thank the paramedics and emergency medical technicians for participating in the study. Our study did not have any sponsors.

Funding This study was financed entirely by the Department of Anaesthesiology of the University Hospital Hamburg.

\section{Competing interests None.}

Ethics approval The local ethics committee (Medical Association Hamburg, Germany) declared that such a study did not require ethical approval. All participants gave written informed consent for their CPR performance data to be evaluated.

Provenance and peer review Not commissioned; externally peer reviewed.

\section{REFERENCES}

1. Handley AJ, Koster R, Monsieurs K, et al. European Resuscitation Guidelines for Resuscitation 2005. Section 2. Adult basic life support and use of automated external defibrillators. Resuscitation 2005;67S1:S7-23.

2. International Liaison Committee on Resuscitation. Consensus on Science and Treatment Recommendations. Part 2: Adult basic life support. Resuscitation 2005;67:187-201.

3. Heilman KM, Muschenheim C. Primary cutaneous tuberculosis resulting from mouth-to-mouth respiration. N Engl J Med 1965;273:1035-6.

4. Christian MD, Loutfy M, McDonald LC, et al. Possible SARS coronavirus transmission during cardiopulmonary resuscitation. Emerg Infect Dis 2004; 10:287-93.

5. Gliwitzky B, Hirsch M, Kohlmann T, et al. Überkopf- versus Standardreanimation: 'Performance contra Zeitgewinn?' Eine prospective Studie am Reanimationsphantom. Rettungsdienst 2001;24:652-7.

6. Wolcke BB, Gliwitzky B, Kohlmann T, et al. Overhead-CPR versus standard-CPR in a two rescuer-ALS-scenario. Resuscitation 2002;55:110.

7. Gliwitzky B, Kohlmann T. "Over head CPR": A sensible method for advanced CPR with two rescue workers? Notfall Rettungsmed 2003;6:193-6.

8. Handley AJ, Handley JA. Performing chest compressions in a confined space. Resuscitation 2004;61:55-61.

9. Perkins GD, Stephenson BT, Smith CM, et al. A comparison between over-the-head and standard cardiopulmonary resuscitation. Resuscitation 2004;61:155-61.

10. Hüpfl M, Duma A, Uray $T$, et al. Over-the-head cardiopulmonary resuscitation improves efficacy in basic life support performed by professional medical personnel with a single rescuer: a simulation study. Anesth Analg 2005;101:200-5.

11. Bollig G, Steen PA, Wik L. Standard versus over-the-head cardiopulmonary resuscitation during simulated advanced life support. Prehosp Emerg Care 2007:11:443-7.

12. Handley AJ, Monsieurs KG, Bossaert LL. European Resuscitation Council Guidelines 2000 for adult basic life support. Resuscitation 2001;48:199-205.

13. Yu T, Weil MH, Tang W, et al. Adverse outcomes of interrupted precordial compression during automated defibrillation. Circulation 2002;106:368-72

14. Kern KB, Sanders AB, Raife J, et al. A study of chest compression rates during cardiopulmonary resuscitation in humans: The importance of rate-directed chest compressions. Arch Intern Med 1992;152:145-9.

15. Abella BS, Alvarado JP, Myklebust $\mathrm{H}$, et al. Quality of cardiopulmonary resuscitation during in-hospital cardiac arrest. JAMA 2005;293:305-10.

16. Wik L, Kramer-Johansen J, Myklebust $\mathrm{H}$, et al. Quality of cardiopulmonary resuscitation during out-of-hospital cardiac arrest. JAMA 2005;293:299-304.

17. Eftestol T, Sunde K, Steen PA. Effects of interrupting precordial compressions on the calculated probability of defibrillation success during out-of-hospital cardiac arrest. Circulation 2002;105:2270-3.

18. Kern KB, Hilwig RW, Berg RA, et al. Importance of continuous chest compressions during cardiopulmonary resuscitation: Improved outcome during a simulated single lay-rescuer scenario. Circulation 2002;105:645-9.

19. Fallaha JF, Spooner BB, Perkins GD. Does dual operator CPR help minimize interruptions in chest compressions? Resuscitation 2009;80:1011-14.

20. Roessler B, Fleischhackl R, Losert $H$, et al. Cardiopulmonary resuscitation and the 2005 universal algorithm: Has the quality of CPR improved? Wien Klin Wochenschr 2009:121:41-6. 